\title{
MITIGATION OF POSITIONING BIAS IN PSI POINT CLOUDS
}

\author{
Sina Montazeri ${ }^{1}$, Fernando Rodríguez González ${ }^{1}$, Xiao Xiang Zhu ${ }^{1,2}$ \\ ${ }^{1}$ Remote Sensing Technology Institute (IMF), German Aerospace Center (DLR) \\ ${ }^{2}$ Signal Processing in Earth Observation (SiPEO), Technical University of Munich (TUM)
}

\begin{abstract}
In this paper, we propose a framework for geocoding error correction of point clouds obtained from Persistent Scatterer Interferometry (PSI) in urban areas. The horizontal positioning bias of Persistent Scatterers (PS) is mitigated by applying geodetic and atmospheric corrections to the PS azimuth and range timings. Furthermore, the vertical positioning bias, caused due to the unknown height of the PSI reference point, is estimated and compensated for by the use of Synthetic Aperture Radar (SAR)-based Ground Control Point (GCP)s. Experimental results based on cross-heading pairs of TerraSAR-X (TS-X) high resolution spotlight images over the city of Berlin, Germany and in Oulu, Finland are presented. For selected test sites, the localization accuracy of the point clouds is analyzed with respect to a reference LiDAR data, which demonstrates the applicability of the proposed correction approach.
\end{abstract}

Index Terms - geocoding, persistent scatterer interferometry, positioning, synthetic aperture radar, TerraSAR-X

\section{INTRODUCTION}

Persistent Scatterer Interferometry (PSI) [1] is a single-master multi-temporal Interferometric Synthetic Aperture Radar (InSAR) technique. It mitigates the effect of decorrelation and atmospheric disturbances by retrieving the height and deformation parameters of only phase-stable targets, known as Persistent Scatterers (PS). In order to present the results of PSI in a common geodetic reference system, a coordinate transformation between the radar datum and an earth-fixed Cartesian reference frame is required, which is called geocoding. The precise knowledge of the master orbit during acquisition as well as PS azimuth and range timings, and the height of PS are necessary input for geocoding. These variables are used to solve the Range-Doppler-Ellipsoid equations [2] to retrieve the three-dimensional (3D) Cartesian coordinates of the PS.

Evidently if any of the parameters involved in geocoding are erroneous, they will affect the localization accuracy of the PS and introduce positioning bias in the generated PSI point cloud. These errors, excluding the effect of orbit inaccuracies that is negligble in modern SAR sensors such as TerraSAR-X (TS-X), include:
- Bias on azimuth and range timings caused by propagation delays, geodynamic impacts and internal sensor effects translating to horizontal positioning offsets.

- Digital Elevation Model (DEM) error of the PSI reference point, which leads to both horizontal and vertical positioning shifts on all PS.

For more information on the magnitude of these errors and their effect on each coordinate component of PS, the reader is referred to [3].

In this paper, we introduce a framework in order to improve the localization accuracy of PSI point clouds by mitigating the above-mentioned biases. We employ SAR imaging geodesy [4] for correcting the radar measurements to increase the positioning accuracy in the horizontal components. We also estimate the DEM error of PSI reference point by using Ground Control Point (GCP)s that are extracted from multiaspect SAR data [5]. In the following the methodology is described and the experimental results on TS-X high resolution spotlight images of Berlin and Oulu are reported.

\section{METHODOLOGY}

The geocoding error correction approach consists of four different parts, which are depicted in the flowchart of Fig. 1. Initially, PSI processing is carried out for the generation of 3D point clouds [6]. Ionospheric and troposheric delays are interpolated for the location and the time of the PS using global Total Electron Content (TEC) maps and numerical weather data, respectively. These corrections are performed using the SAR Geodesy Processor (SGP) of the German Aerospace Center (DLR) [7] and are subtracted from the radar timings to provide absolute azimuth and range times for each PS.

The extraction of SAR-based GCPs requires a minimum of two SAR image stacks acquired from separate orbits. The procedure detects identical PS from different viewing geometries, extracts and calibrates their radar timings and estimates the absolute 3D position of the PS through stereo SAR [8]. At the final stage of the framework, the generated GCPs from the previous step are matched with their corresponding PS in the PSI point clouds. Subsequently, a histogram is formed by evaluating the difference between the ellipsoidal heights of the PS and the GCPs. The mode of the histogram is then 


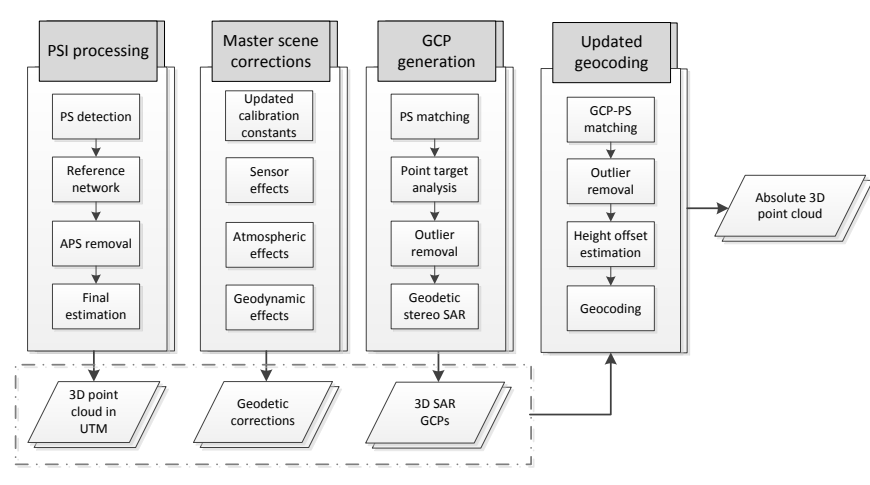

Fig. 1: The flowchart of the geocoding error correction approach. The double shapes imply that at least two stacks of SAR images from different viewing geometries are required.

estimated, which represents the constant DEM error of the reference point. The retrieved height offset is subtracted from the height values of all the PS and along with the absolute timing measurements, an updated geocoding is carried out. The result includes the new corrected PSI point clouds with improved localization accuracy. For more details on each step of the geocoding correction framework, the reader is referred to [3].

\section{EXPERIMENTAL RESULTS}

In this Section, the results of applying the introduced geocoding correction scheme is reported on two data sets over Berlin and Oulu. The localization accuracy of the obtained absolute PSI point clouds is analyzed with respect to a highly accurate LiDAR data for selected test sites in Berlin. Furthermore, preliminary results are shown for geodetic point cloud fusion, which allows for the coregistration of multi-aspect point clouds in order to achieve absolute shadow-free PSI products.

\subsection{Data Description}

The SAR images used in this study are all from the high resolution spotlight mode of TS-X with range bandwidth of 300 MHz. Berlin data set includes a pair of cross-heading stacks with a total number of 214 images. The Oulu data set consists of four stacks, two acquired from descending orbits and two from ascending ones including 177 images. Complementary to the SAR data, for Berlin a LiDAR point cloud with localization accuracy better than $10 \mathrm{~cm}$ is available, which is used as reference for positioning accuracy evaluation.

\subsection{Data Processing}

The PSI processing has been carried out by the PSI-GENESIS of DLR [6, 9]. For each PS, updated topography, linear deformation rate and the amplitude of seasonal motion were con-
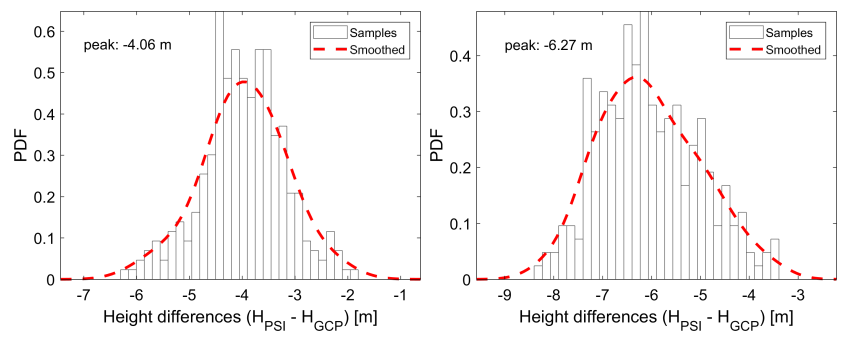

Fig. 2: The height difference histograms among GCPs and their corresponding PS for two stacks in Berlin. The peak of the smoothed histograms denote the estimated DEM error of respective reference points.

sidered. The latter is necessary to model since the data sets include X-band SAR images of urban areas and span a few years. The required output from PSI processing is the 3D PSI point cloud in the UTM coordinate system.

The geodetic and geodynamic corrections were performed using SGP [7]. The TS-X geometric calibration constants were also updated according to the recent results from long term corner reflector campaigns [10]. The average of sum of all corrections in range and azimuth, for both Berlin and Oulu data sets, amounted to almost three meters and less than one decimeter, respectively. These corrections were subtracted from the original timing measurements to provide unbiased timings for each PS.

The generation of GCPs for Oulu and Berlin were carried out based on the methods described in [5] and in [11], respectively. A filtering based on the posterior standard deviations of Stereo SAR was done, which resulted in approximately 1000 GCPs distributed through the entire extend of PSI point clouds. All GCPs have precision values better than $10 \mathrm{~cm}$ in all coordinate components. Previous experiments have reported an absolute accuracy better than $20 \mathrm{~cm}$ for selected GCPs [11].

The final part of the framework is concerned with matching the GCPs with their corresponding PS in the PSI point clouds and robustly estimating the DEM error of reference points of each point cloud. For this, the nearest neighbor of the GCPs with an amplitude dispersion index lower than 0.4 is selected and ellipsoidal height difference histograms are formed. The height differences for Berlin are depicted in Fig. 2 where DEM errors of $-4.06 \mathrm{~m}$ and $-6.27 \mathrm{~m}$ are estimated after smoothing the histograms. These values are used to calibrate PS heights and along with the corrected range and azimuth timings, the geocoding is repeated to obtain absolute 3D PSI point clouds.

\subsection{Cross-Comparison with LiDAR}

For a few test sites in the Berlin case study, positioning accuracy of the corrected PSI point clouds is analyzed with respect to a reference LiDAR data. In order to make the PSI 

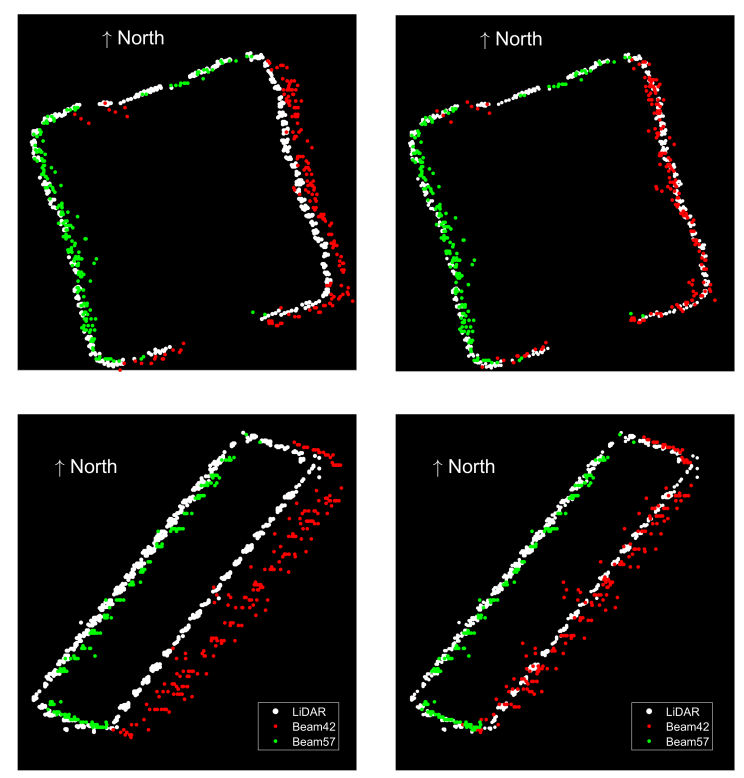

Fig. 3: Horizontal localization accuracy of descending (red) and ascending (green) point clouds with respect to LiDAR (white). The figures in left and right column depict the PSI point clouds before and after correction, respectively. The correction causes the point clouds to be aligned with the LiDAR data.

point clouds more compatible with the LiDAR data, PS from facade of buildings are excluded for evaluating the vertical localization accuracy. To this end, within a neighborhood of $4 \mathrm{~m}$ around each point, the variance of ellipsoidal heights is estimated. If the variance is higher than $1.5 \mathrm{~m}$, the point will be considered as a facade PS and will be excluded. The difference between the height histograms of LiDAR and the PSI point clouds then denote the absolute vertical accuracy. For analyzing the horizontal accuracy, the same approach is used to select the facade PS from point clouds. The distance of these PS are then calculated with respect to the corresponding building footprint from LiDAR, to evaluate the absolute horizontal accuracy.

For a few test sites in Berlin, the results of the aforementioned procedure for corrected and non-corrected PSI results can be seen in Fig. 3 for horizontal analysis and in Fig. 4 for the vertical analysis, respectively. In all figures, the white dots represent the LiDAR point cloud while the red and green dots denote the descending and ascending point clouds. It is seen that after the corrections, the point clouds move closer to the LiDAR data. The mean distance of the non-corrected point clouds is about $2.5 \mathrm{~m}$ before applying the corrections while this value is improved to $40 \mathrm{~cm}$ for the corrected point clouds. The absolute height accuracy for the ascending track of Berlin, seen for a test site in Fig. 4, is about $4.3 \mathrm{~m}$ before correction and around $20 \mathrm{~cm}$ after applying the correction scheme.

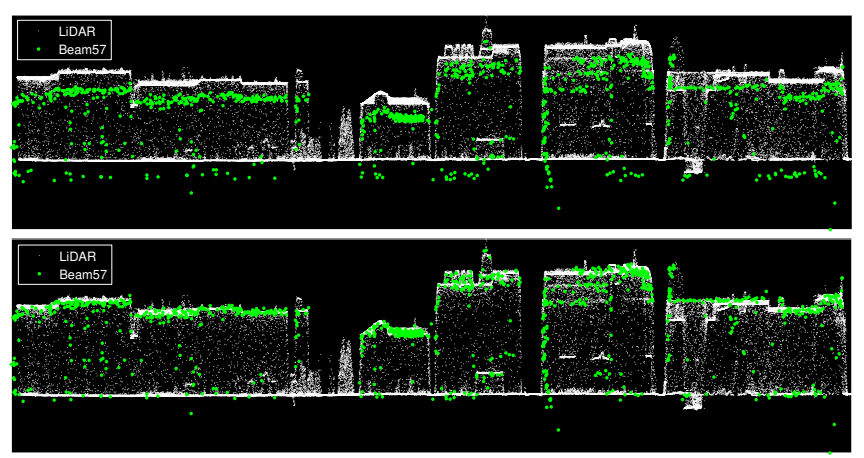

Fig. 4: Vertical accuracy analysis of the ascending PSI point cloud (green) with respect to LiDAR (white) before (top) and after (bottom) applying the corrections. Corrections compensate for the vertical shift apparent in the top figure.

\subsection{Geodetic Point Cloud Fusion}

The availability of multiple absolute PSI point clouds from different viewing geometries allows for the reconstruction of shadow-free detailed point clouds with high localization accuracy. Since for the Oulu data set no reference data is available for evaluating the positioning accuracy of the generated point clouds, we visually inspect the results of point cloud fusion before and after applying the introduced geocoding correction method. Fig. 5 shows the the full $2 \mathrm{D}$ view of the fusion of two ascending and two descending point clouds over Oulu color-coded according to height. The total number of scatterers is approximately seven million in an area of $50 \mathrm{~km}^{2}$.

For a test site, the 2D alignment of one ascending and one descending point cloud of Oulu is checked in Fig. 6. The ascending and descending point clouds are visualized as green and red dots, respectively. It is clear in the left figure that the end point of buildings captured from opposite sides do not match. This horizontal offset, which on average is approximately $4.7 \mathrm{~m}$ in East and almost $1.8 \mathrm{~m}$ in North, is largely mitigated after performing geodetic point cloud fusion in the right figure. This indicates the relative correctness of the fusion algorithm. As the final example, Fig. 7 shows an overlay of the non-corrected (left) and the corrected (right) PSI point clouds for a small test site in Oulu. It is observed that after the geodetic point cloud fusion, the point clouds are aligned with the corresponding side of the buildings seen in the aerial ortho-photos.

\section{CONCLUSION}

In this paper, we proposed a framework for mitigation of positioning bias in PSI point clouds. The method does not use any auxiliary positioning data for the calibrations and can be performed as a post-processing step after the generation of PSI point clouds. The PSI products with enhanced localization accuracy allow the association of scatterers to their true 


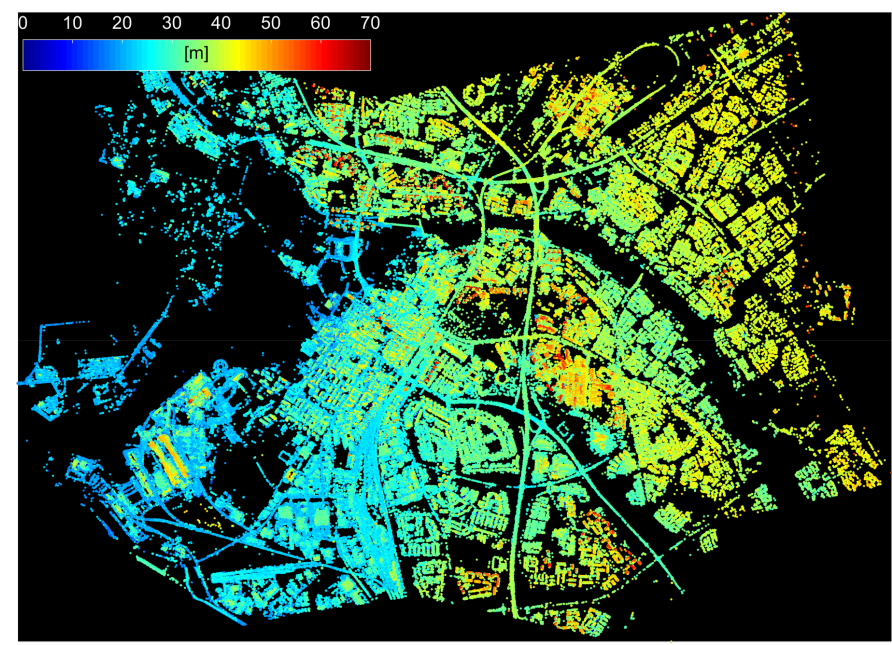

Fig. 5: Geodetic fusion of four PSI point clouds of Oulu. The ellipsoidal height of the point clouds is color-coded.
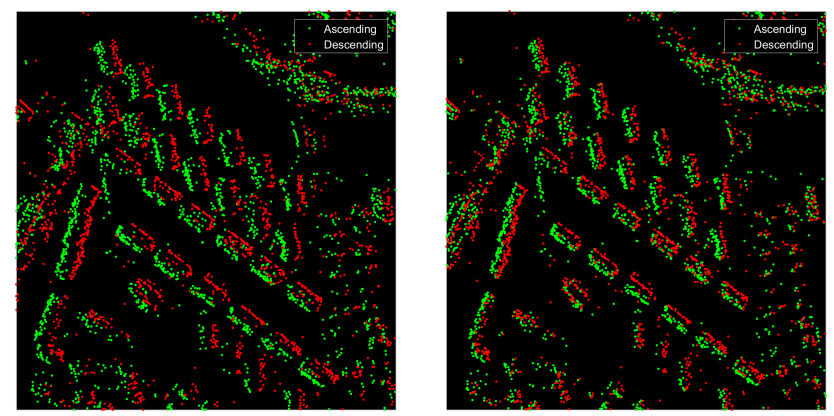

Fig. 6: A 2D view (East-North plane) on one ascending and one descending point cloud of Oulu before (left) and after (right) applying the proposed corrections.
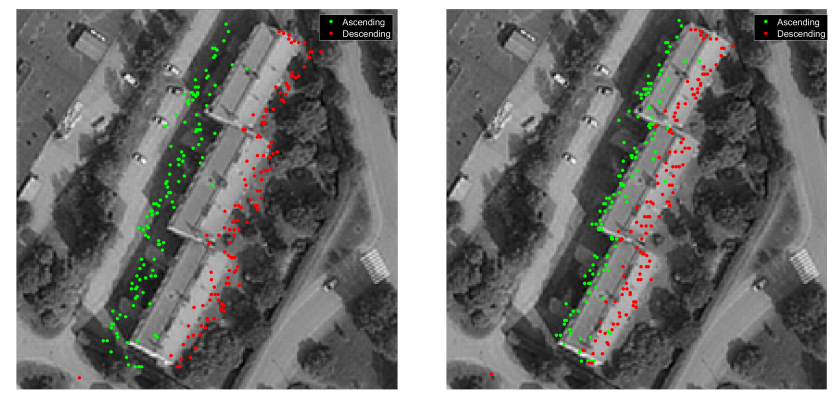

Fig. 7: Ascending and descending point clouds of Oulu overlaid on the corresponding aerial ortho-photo before (left) and after (right) applying the geocoding correction approach. The results after correction allow for the assignment of the PS to its corresponding building parts.

positions. This is in particular important when dealing with high resolution SAR products and where small-scale deformation monitoring of individual objects is required. Apart from these, the improvement in the positioing accuracy of PSI point clouds allows for easier comparison and integration of the point clouds to data from other sensors.

\section{ACKNOWLEDGEMENT}

The authors would like to thank Dr. Ulrich Balss for his technical support on the GCP generation part of the proposed methodology. We are also grateful to Dr. Heiko Hirschmüller of the DLR robotics institute for providing us with the optical data of Berlin. The LiDAR data of Berlin have been provided by Land Berlin (EU EFRE project) and Landesamt für Vermessung und Geoinformation Bayern.

\section{REFERENCES}

[1] A. Ferretti, C. Prati, and F. Rocca, "Permanent scatterers in SAR interferometry," IEEE Transactions on Geoscience and Remote Sensing, vol. 39, no. 1, pp. 8-20, 12001.

[2] M. Schwabisch, "A fast and efficient technique for SAR interferogram geocoding," in Geoscience and Remote Sensing Symposium (IGARSS). 1998, pp. 1100-1102 vol.2, IEEE.

[3] S. Montazeri, F. Rodríguez González, and X.X. Zhu, "Geocoding Error Correction for InSAR Point Clouds," Remote Sensing, vol. 10, no. 10, pp. 1523, Sept. 2018.

[4] M. Eineder, C. Minet, P. Steigenberger, X.Y. Cong, and T. Fritz, "Imaging Geodesy - Toward Centimeter-Level Ranging Accuracy With TerraSAR-X," IEEE Transactions on Geoscience and Remote Sensing, vol. 49, no. 2, pp. 661-671, 2 2011.

[5] S. Montazeri, C. Gisinger, M. Eineder, and X.X. Zhu, "Automatic Detection and Positioning of Ground Control Points Using TerraSAR-X Multiaspect Acquisitions,' IEEE Transactions on Geoscience and Remote Sensing, pp. 1-20, 2018.

[6] N. Adam, F. Rodriguez Gonzalez, A. Parizzi, and R. Brcic, "Wide area Persistent Scatterer Interferometry: Current developments, algorithms and examples," in Geoscience and Remote Sensing Symposium (IGARSS). 7 2013, pp. 1857-1860, IEEE.

[7] M. Eineder, U. Balss, S. Suchandt, C. Gisinger, X. Cong, and H. Runge, "A definition of next-generation SAR products for geodetic applications," in Geoscience and Remote Sensing Symposium (IGARSS). 7 2015, pp. 1638-1641, IEEE.

[8] C. Gisinger, U. Balss, R. Pail, X.X. Zhu, S. Montazeri, S. Gernhardt, and M. Eineder, "Precise Three-Dimensional Stereo Localization of Corner Reflectors and Persistent Scatterers With TerraSAR-X," IEEE Transactions on Geoscience and Remote Sensing, vol. 53, no. 4, pp. 1782-1802, 42015.

[9] B.M. Kampes, Radar interferometry: persistent scatterer technique, Number v. 12 in Remote sensing and digital image processing. Springer, Dordrecht, the Netherlands, 2006.

[10] U. Balss, C. Gisinger, and M. Eineder, "Measurements on the Absolute 2-D and 3-D Localization Accuracy of TerraSAR-X," Remote Sensing, vol. 10, no. 4, pp. 656, 42018.

[11] U. Balss, H. Runge, S. Suchandt, and X.Y. Cong, "Automated extraction of 3-D Ground Control Points from SAR images an upcoming novel data product," in Geoscience and Remote Sensing Symposium (IGARSS). 7 2016, pp. 5023-5026, IEEE. 